\title{
SoC-approach for safety-oriented multi-channel communication in industrial application of human- robot-collaboration (HRC)
}

\author{
Mohamed Abdelawwad \\ ICAS, Institute for Computer \\ Architecture and System Programming \\ University of Kassel \\ Kassel, Germany \\ m.abdelawwad@uni-kassel.de
}

\author{
Michael Schwarz \\ ICAS, Institute for Computer \\ Architecture and System Programming \\ University of Kassel \\ Kassel, Germany \\ m.schwarz@uni-kassel.de
}

\author{
Malte Drabesch \\ ICAS, Institute for Computer \\ Architecture and System Programming \\ University of Kassel \\ Kassel, Germany \\ malte.drabesch@uni-kassel.de \\ Josef Börcsök \\ ICAS, Institute for Computer \\ Architecture and System Programming \\ University of Kassel \\ Kassel, Germany \\ j.boercsoek@uni-kassel.de
}

\author{
Christian Voss \\ ICAS, Institute for Computer \\ Architecture and System Programming \\ University of Kassel \\ Kassel, Germany
}

\begin{abstract}
The importance of human-robot collaboration systems in industrial applications has increased due to the orientation towards the smart factory approach. To ensure the safety of such systems, collision avoidance techniques based on millimeter-wave radar sensors are used. Safe communication between the robot and the radar sensor is ensured by using the safety system on a chip based on 10o2D architecture. The design and prototyping of a multi-channel communication FPGA-based development board is presented. Furthermore, the FPGA implementation of SoC with multiple Ethernet MAC interfaces based on various soft cores is demonstrated.
\end{abstract}

Keywords-Safety System on a Chip, 1oo2D Safety Architecture, Human-Robot Collaboration

\section{INTRODUCTION}

Industry 4.0 is the way to the future of the industrial environment through the development of the so-called smart factories [1]. In this new approach, the connection between the physical world and the virtual world is established using Cyber-Physical Systems (CPSs), Internet of Things (IoT) and Internet of Services (IoS) [2]. The integration of CPSs into the industrial environment results in the Cyber-Physical Production Systems (CPPSs) that increases the flexibility and productivity of the production systems [3, 4]. The development of Industry 4.0 concept is based on the development of nine technology sectors, i.e., internet of things, cybersecurity, cloud computing, big data analytics, simulation, additive manufacturing, augmented reality, artificial intelligence and autonomous robots $[5,6]$.

Robotics in the industrial environment is a cornerstone that helps to realize the industry 4.0 concept. This concept includes the integration of free movement robotic systems, which provides flexibility and also increases the throughput. However, the mechanical force imparted by the industrial robotics is enormous. Hence, the presence of operators and other personal staff in the same working area may increase the risk of injury or death in case of malfunctioning of the robot or personnel in the vicinity of the robots while it is in operation. Therefore, in order to ensure safety in the industrial environment, the industrial standards EN ISO 12100 [7], EN ISO 10218 [8] and ISO/TS 15066 [9] regulates the safety requirements for machinery, robotics and human-robot collaboration, respectively. Based on the ISO/TS 15066, the force, speed and boundary of the working area of the industrial robots in the presence of human personnel are restricted. There are four approaches of HRC, i.e., Safety-rated monitored stop, Hand-guided, Speed \& Separation Monitoring, Power \& Force Limiting [10]. For example, the robotic systems are confined in a cage with interlock functionality. As an operator enters the operation area of the robot, it halts its operation. Such a situation hinders the throughput and leads to production overload. The restrictions mentioned above in the operation mode limits the flexibility and increases the cost and the infrastructural complexity of the production lines.

The concept of human-robot-collaboration is successfully implemented using various collision avoidance techniques. Projection-based sensor systems [11], vision-based systems [12] natural communication-based systems [13], capacitive skin sensor-based systems [14], ultrasonic based systems [15] are examples of HRC approaches. The millimeter-wave radar sensor has a potential on realizing HRC systems due to their small size and lightweight [16]. Compared to the other collision avoidance techniques, millimeter-wave radar sensors-based systems are a good match to the industrial applications. The reason for this is the presence of aggressive particles and vapor that degrades the optical-based collision avoidance system in the industrial environments [17]. Within the scope of a funded project by BMBF [18], a smart approach is implemented based on using multiple millimeterwave radar sensors. The principle safety architecture of human-robot-collaboration system is shown in Fig. 1.

However, to implement this concept, the foundation hardware is required that enables the HRC. Additionally, the hardware must be functionally safe to achieve this goal and to qualify as per the industrial standard. To establish high reliability and safety of the system, a new FPGA based communication SoC is designed on 10o2D architecture [1921]. In this paper, the design and integration of the used development board are introduced while the safety architecture and its FPGA implementations are described in reference [22].

\section{SOC ARCHITECTURE FOR SAFETY SENSOR NODE}

The realization of current project enables in expanding to other innovative ideas of direct HRC in a physically close area. This technology can be adapted to produce various applications in the field of personal and home environment, 
health and industrial. The system consists of 16 radar sensor nodes, a safety System-on-Chip (SoC), a data evaluation board and communication interfaces. The radar sensor nodes are distributed on a ring that surrounds the robotic arm. Each radar sensor node covers a detection angle of $60^{\circ}$. With a certain degree of overlap among the sensor nodes, a total of 8 radar sensor nodes cover the complete 3D-detection range of $360^{\circ}$. The remaining eight radar sensors nodes provide redundancy to the detection subsystem. The data collected by the radar sensor nodes is processed by the FPGA based safety SoC. The redundancy principle of the system and the architecture of Safety SoC are discussed in ref. [22]. The radar sensor nodes are connected to the SoC via the fast
Ethernet that provides a data speed of up to $100 \mathrm{Mbps}$. The SoC checks the validity of the collected data and transmits it to the evaluation hardware via a gigabit Ethernet communication interface. The data evaluation board validates and processes this data. The processing of the data includes calculating the number of objects around the robot arm, the direction of movement and the speed. Based on this information and the position and the speed information provided by the robot, a decision is made to continue the operation, reduces the speed else the robot makes a transition into a predefined safe state. The transition to the safe state is attained when a collision with any object cannot be avoided.

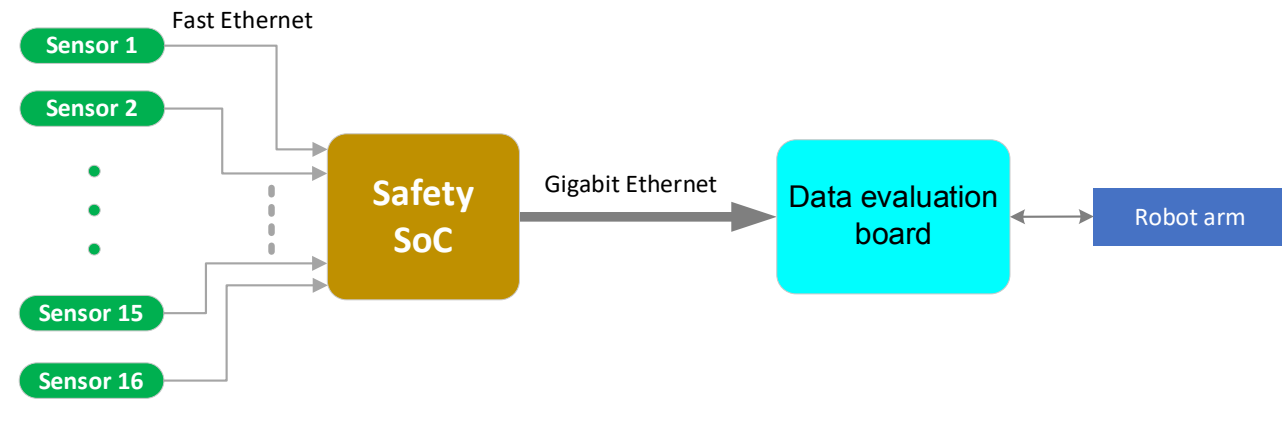

Fig. 1: Principle safety architecture of human-robot-collaboration system

\section{ARCHITECTURAL DESIGN OF SAFETY SOC}

A novel FPGA based safety SoC is developed within the scope of this project that supports multi-channel communication over Ethernet (17 ports). Therefore, a development board with multiple Ethernet ports was a prerequisite. However, such a development board is not available in the market. In this section, the development process of the in-house FPGA development board is described to satisfy the requirements for the current application.

\section{A. Methods and measures for implementation and testing of SoC design}

The standard Virtex 7 FPGA development board supports the development of hardware design for embedded systems. As a requirement for the application of current project, it could not qualify due to the fact that it provides just a single Ethernet port without additional add-ons to the board. The number of Ethernet ports is extended by attaching Gigabit Ethernet FPGA Mezzanine Card (FMC), from Opsero Electronic Design Inc., to the Virtex 7 development board. A single FMC has four Gigabit Ethernet ports and a maximum of two FMCs are possible to connect to a Virtex 7 board. This Ethernet configuration extends to a total of 9 ports.

For testing purposes, 10o1 SoC with nine Gigabit Ethernet interfaces based on MicroBlaze soft processor is implemented. The Gigabit Ethernet interfaces of implemented design is tested using the Xilinix SDK. Additionally, the embedded Linux OS based PetaLinux is customized, configured and built for the target development board [23, 24]. All nine Gigabit Ethernet interfaces are successfully implemented and tested for transmission.
However, the number of Ethernet interfaces still falls short for our application. Due to this limitation in the number of Ethernet ports, the Virtex 7 board with the FMCs does not satisfy the requirement for this application. Hence, it becomes essential to develop an in-house FPGA development board.

To develop the in-house FPGA development board, the first step was the selection of the design from the two available approaches. One is designing the board based on FPGA chip and the second is to use an FPGA module. The FPGA chip requires designing the complete circuitry, which is complex and time-consuming. In contrast, the FPGA module can be readily integrated onto the FPGA baseboard for design and testing. As soon as the SoC is tested, the inhouse FPGA board based on the FPGA module can be easily developed. Therefore, the second approach was selected.

The Virtex 7 used in the testing phase is available in the market as a chip but not as a module. This scarcity was fulfilled by the Kintex module of the FPGA family. The used FPGA module is Mercury+ KX2 board from Enclustra FPGA Solutions. Also, the baseboard Mercury+ PE1 with two FMC connectors is used to test the combination of the soft core, FPGA module and the Gigabit Ethernet FMC cards. As a final implementation of the SoC for this project, the LEON3 processor is used. The LEON3 soft processor is a modular and configurable VHDL-based 32-bit processor [25].

A 1oo1 LEON3 based SoC with ten Gigabit Ethernet interfaces is implemented on the Mercury+ KX2 FPGA module and tested along with the Mercury+ PE1 baseboard and the Gigabit Ethernet FMC cards. Since the Ethernet interfaces of sensor nodes support only $100 \mathrm{Mb}$ Ethernet 
communication, additional implementation of $100 \mathrm{Mb}$ Ethernet interfaces on the LEON3 SoC is performed and tested using the GPIO extension connector in the Mercury+ PE1 baseboard.

\section{B. Fundamental design of multi-channel communication system}

In the final stage, the multi-channel Ethernet development board is designed and realized. The development board has sixteen Ethernet ports each of 100 $\mathrm{Mb}$ and one Gigabit Ethernet port. Multiple UART communication interfaces and flash memory chips are also integrated. In comparison to the standard solutions (Virtex 7 development board and Mercury KX2 board), the in-house multi-channel Ethernet development board has many advantages (Fig. 2). The number of Ethernet ports is higher and the size of the board is smaller compared to the standard development boards.

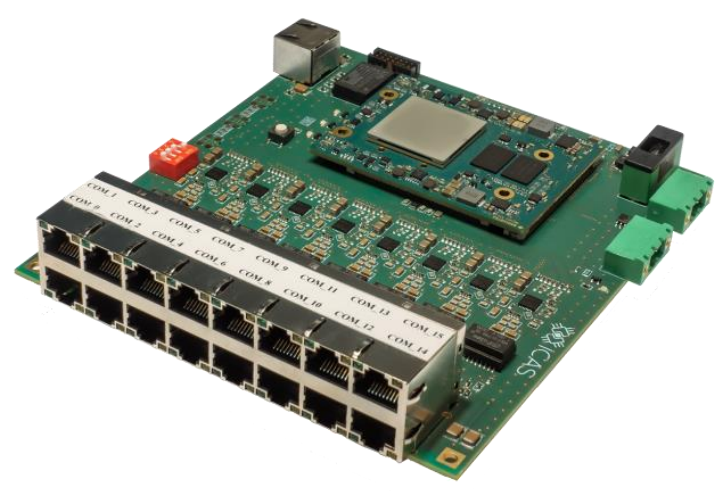

Fig. 2: The in-house multi-channel communication system.

\section{Application of multi-channel communication system}

The implementation of the SoC is carried out and tested in the laboratory. Furthermore, a demonstrator was built with the partners of this project to integrate and test the overall system, which was successfully validated (Fig. 3).

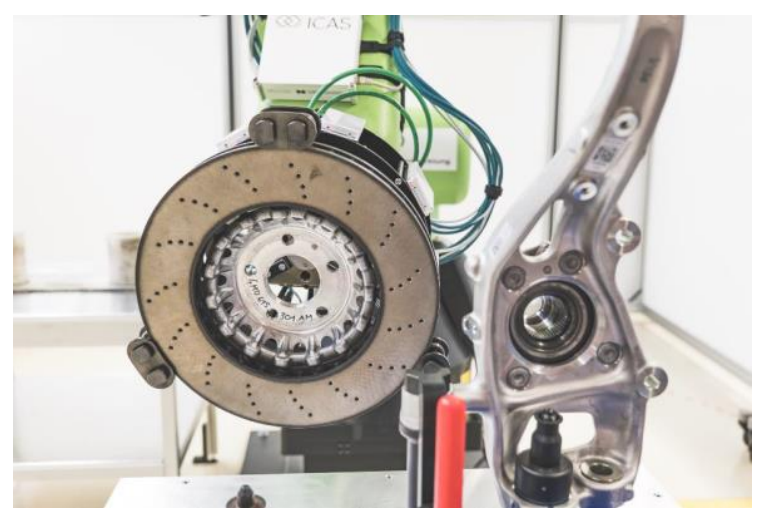

Fig. 3: Demonstrator of RoKoRa project.

\section{CONCLUSION}

In this paper, a smart approach of human-robot collaboration is introduced. Various standard development boards have been tested by implementing multiple Ethernet mac interfaces based on Microblaze and LEON3 soft processors. The implementation has been carried out using various FPGA families, i.e., Virtex 7, Artix 7 and Kintex 7. In the end, the in-house multi-channel communication FPGA based development board is developed to be used on the implementation of the Safety SoC. Verification and validation of the in-house multi-channel communication development board have been successfully conducted. In future work, a safety SoC based on 10o2D safety architecture will be presented.

\section{ACKNOWLEDGEMENTS}

We acknowledge Federal Ministry of Education and Research (BMBF) for financial support (Project number 16SV7815). We acknowledge our colleagues and co-workers at the Department of Computer Architecture and System Programming, University of Kassel for their support, comments, and discussion. Also we acknowledge our project partners for the productive and efficient collaboration, i.e., Fraunhofer-IAF, FANUC Robotics GmbH, AUDI AG, IMST GmbH, German Social Accident Insurance (DGUV), and Fraunhofer-SCAI.

\section{REFERENCES}

[1] S. EL HAMDI and A. ABOUABDELLAH, "Proposal of a General Framework of Smart Factory," in 2020 International Conference on Electrical and Information Technologies (ICEIT), Rabat, Morocco, Mar. 2020 - Mar. 2020, pp. 1-6.

[2] D. Sinha and R. Roy, "Re-viewing CPS as a Part of Smart Factory in Industry 4.0," IEEE Engineering Management Review, p. 1, 2020.

[3] F. Pérez, E. Irisarri, D. Orive, M. Marcos, and E. Estevez, "A CPPS Architecture approach for Industry 4.0," in 2015 IEEE 20th Conference on Emerging Technologies \& Factory Automation (ETFA), 2015, pp. $1-4$.

[4] K. Zhou, Taigang Liu, and Lifeng Zhou, "Industry 4.0: Towards future industrial opportunities and challenges," in 2015 12th International Conference on Fuzzy Systems and Knowledge Discovery (FSKD), 2015, pp. 2147-2152.

[5] M. Rüßmann et al., "Industry 4.0: The future of productivity and growth in manufacturing industries," Boston Consulting Group, vol. 9, no. 1, pp. 54-89, 2015.

[6] F. K. Konstantindis, A. Gasteratos, and S. G. Mouroutsos, "Vision-Based Product Tracking Method for Cyber-Physical Production Systems in Industry 4.0," in 2018 IEEE International Conference on Imaging Systems and Techniques (IST), 2018, pp. 1-6.

[7] DIN EN ISO 12100:2011-03, Sicherheit von Maschinen_- Allgemeine Gestaltungsleitsätze_Risikobeurteilung und Risikominderung (ISO_12100:2010); Deutsche Fassung EN_ISO_12100:2010, Berlin.

[8] DIN EN ISO 10218-1:2012-01, Industrieroboter_Sicherheitsanforderungen_-Teil_1: Roboter 
(ISO_10218-1:2011); Deutsche Fassung EN_ISO_10218-1:2011, Berlin.

[9] DIN ISO/TS 15066:2017-04, Roboter und Robotikgeräte_- Kollaborierende Roboter (ISO/TS_15066:2016), Berlin.

[10] M. J. Rosenstrauch and J. Kruger, "Safe human-robotcollaboration-introduction and experiment using ISO/TS 15066," in 2017 3rd International Conference on Control, Automation and Robotics (ICCAR), Nagoya, Japan, Apr. 2017 - Apr. 2017, pp. 740-744.

[11] C. Vogel, C. Walter, and N. Elkmann, "A projectionbased sensor system for safe physical human-robot collaboration," in 2013 IEEE/RSJ International Conference on Intelligent Robots and Systems, Tokyo, Nov. 2013 - Nov. 2013, pp. 5359-5364.

[12] F. Flacco, T. Kroger, A. de Luca, and O. Khatib, "A depth space approach to human-robot collision avoidance," in 2012 IEEE International Conference on Robotics and Automation, Saint Paul, MN, May. 2012 May. 2012, pp. 338-345.

[13] I. Maurtua, I. Fernandez, J. Kildal, L. Susperregi, A. Tellaeche, and A. Ibarguren, "Enhancing safe humanrobot collaboration through natural multimodal communication," in 2016 IEEE 21st International Conference on Emerging Technologies and Factory Automation (ETFA), Berlin, Germany, Sep. 2016 - Sep. 2016, pp. 1-8.

[14] K. M'Colo, B. Luong, A. Crosnier, C. Néel, and P. Fraisse, "Obstacle Avoidance using a Capacitive Skin for Safe Human-Robot Interaction," in 2019 IEEE/RSJ International Conference on Intelligent Robots and Systems (IROS), 2019, pp. 6742-6747.

[15] M. J. Rosenstrauch and J. Kruger, "Safe human robot collaboration - Operation area segmentation for dynamic adjustable distance monitoring," in 2018 th International Conference on Control, Automation and Robotics (ICCAR), Auckland, Apr. 2018 - Apr. 2018, pp. 17-21.
[16] T. Geibig, A. Shoykhetbrod, A. Hommes, R. Herschel, and N. Pohl, "Compact 3D imaging radar based on FMCW driven frequency-scanning antennas," in 2016 IEEE Radar Conference (RadarConf), Philadelphia, PA, USA, May. 2016 - May. 2016, pp. 1-5.

[17] M. van Delden, C. Guzy, and T. Musch, "Investigation on a System for Positioning of Industrial Robots Based on Ultra-Broadband Millimeter Wave FMCW Radar," in 2019 IEEE Asia-Pacific Microwave Conference (APMC), 2019, pp. 744-746.

[18] RoKoRa - Mensch-Technik-Interaktion. [Online]. Available: https://www.technik-zum-menschenbringen.de/projekte/rokora (accessed: Jun. 19 2020).

[19] M. H. Schwarz and J. Börcsök, "Reliable system design with a high degree of diagnostic procedures for embedded systems," in 24th IET Irish Signals and Systems Conference (ISSC 2013), 2013, pp. 1-7.

[20] A. Hayek, B. Machmur, M. Schreiber, and J. Börcsök, "Embedded safety on a chip for SIL3 applications," in 2014 26th International Conference on Microelectronics (ICM), 2014, pp. 100-103.

[21] J. Börcsök, Functional Safety: Basic Principles of Safety-related Systems, 2nd ed. Berlin: VDE Verlag, 2017.

[22] M. Abdelawwad, M. Drabesh, M. Schwarz, Josef Börcsök, "Ethernet communication SoC based on 10o2D architecture for safety industrial human-robotcollaboration," Under submission.

[23] Multi-port Ethernet in PetaLinux. [Online]. Available: https://www.fpgadeveloper.com/2016/05/multi-portethernet-in-petalinux.html (accessed: Jun. 19 2020).

[24] PetaLinux Tools. [Online]. Available: https:// www.xilinx.com/products/design-tools/embeddedsoftware/petalinux-sdk.html (accessed: Jun. 19 2020).

[25] Cobham Gaisler AB, LEON3. [Online]. Available: https://www.gaisler.com/index.php/products/ processors/leon3 (accessed: Jun. 19 2020).

\section{Creative Commons Attribution License 4.0 (Attribution 4.0 International, CC BY 4.0)}

This article is published under the terms of the Creative Commons Attribution License 4.0 https://creativecommons.org/licenses/by/4.0/deed.en_US 